\title{
Downstaging of Recurrent Advanced Hepatocellular Carcinoma After Lenvatinib Treatment: Opportunities or Pitfalls? A Case Report
}

This article was published in the following Dove Press journal: OncoTargets and Therapy

\section{Zhaobo Liu (D) \\ Zhi Fu (D) \\ Guangming $\mathrm{Li}$ \\ Dongdong Lin (iD)}

Department of General Surgery, Beijing Youan Hospital, Capital Medical University, Beijing 100069, People's Republic of China
Correspondence: Guangming Li

Department of General Surgery, Beijing

Youan Hospital, Capital Medical

University, Beijing 100069, People's

Republic of China

Email liguangming@ccmu.edu.cn

Dongdong Lin

Department of General Surgery, Beijing

Youan Hospital, Capital Medical

University, Beijing 100069, People's

Republic of China

Tel +86-10-83997466

Fax +86-|0-6329337|

Email IddI23I@ccmu.edu.cn
Background: The majority of patients with hepatocellular carcinoma (HCC) are diagnosed at an advanced stage that excludes them from potentially curative surgical treatment. Lenvatinib is associated with a high objective response rate (ORR) (40.6\%) in advanced $\mathrm{HCC}$, indicating the potential for tumor downstaging and conversion to surgical intervention. We report the case of a patient with recurrent, advanced HCC who achieved a partial response and downstaging following third-line treatment with lenvatinib but missed the opportunity for conversion hepatectomy.

Case Presentation: A male Chinese patient aged 42 years presented with an obstructive liver lesion, revealed by CT imaging to be a single tumor in segments V and VIII of the liver, without macrovascular invasion. The patient had chronic hepatitis B infection, Barcelona Clinic Liver Cancer (BCLC) Stage A, normal liver function (Child-Pugh Score 5 and Grade A) and AFP level $4.45 \mathrm{ng} / \mathrm{mL}$. The patient underwent a successful hepatectomy but experienced recurrence 14 months later. The recurrent tumor was detected at an early stage and the patient underwent successful radiofrequency ablation and transarterial chemoembolization. After a further 11 months, the patient experienced a second relapse characterized by multiple disseminated metastases in the left and right lobes of the liver and possible macrovascular invasion, equal to BCLC Stage B/C. The patient received lenvatinib and achieved a partial response with complete disappearance of a number of lesions, recovering to BCLC Stage A and becoming eligible for liver transplantation. However, the patient refused surgery and after 4 months experienced progressive disease.

Conclusion: Our case suggests that radical treatment, such as conversion hepatectomy or liver transplantation, should be undertaken quickly following downstaging and within the expected PFS time associated with lenvatinib. However, further studies are required to provide additional evidence for this treatment strategy.

Keywords: hepatocellular carcinoma, lenvatinib, conversion hepatectomy, liver transplantation

\section{Background}

Primary liver cancer is the seventh most common cancer and represents the third highest cause of cancer-related death. ${ }^{1}$ Of the primary liver cancers, hepatocellular carcinoma (HCC) is the most prevalent, accounting for $75-85 \%$ of all cases. ${ }^{1}$ For patients with HCC, surgery is the only potentially curative treatment option and in 
large retrospective studies has been associated with 5-year overall survival rates $>50 \%{ }^{2-6}$ In contrast, patients with unresectable $\mathrm{HCC}$ have 5-year overall survival rates of approximately $2-11 \%{ }^{7}$ Based on the recommendations of most international treatment guidelines, only around 5 $-10 \%$ of patients with $\mathrm{HCC}$ are considered suitable for surgical resection, in many cases because progression beyond early-stage disease has already occurred by the time of diagnosis. ${ }^{3,8}$ Therefore, treatment strategies to downstage patients with moderate to advanced HCC and enable them to be considered suitable for surgical resection or liver transplantation would have the potential to benefit a large number of patients.

For patients with HCC considered ineligible for surgical intervention, locoregional and systemic therapy are the primary recommended treatments. ${ }^{9-11}$ First-line systemic therapy options for advanced HCC include sorafenib (an oral inhibitor of vascular endothelial growth factor receptor (VEGFR) 1-3, platelet-derived growth factor receptor (PDGFR)- $\beta$, Raf, Flt-3, and KIT) and lenvatinib (an oral inhibitor of VEGFR 1-3, fibroblast growth factor receptor (FGFR) 1-4, PDGFR, RET and KIT). ${ }^{9-11}$ Second-line options include immunotherapy, regorafenib, cabozantinib and ramucirumab. The pivotal Phase III trials of sorafenib and lenvatinib in HCC focused mainly on overall survival. $^{12,13}$ However, the Phase III trial of lenvatinib reported very high objective response rates (ORR; $40.6 \%$; 95\% CI, 36.2-45.0) ${ }^{14}$ and in a Phase I study the combination of lenvatinib with an immune checkpoint inhibitor was also associated with high response rates $(36.7 \%){ }^{14-16}$ These results indicate that a large proportion of patients receiving lenvatinib-based systemic therapy will experience a decreased tumor burden and may achieve tumor downstaging, providing the potential for successful surgical resection.

Previous case reports have described patients with unresectable HCC achieving a complete response to combined lenvatinib and immune therapy, ${ }^{15}$ and downstaging followed by successful resection following transarterial chemoembolization (TACE) ${ }^{17}$ and combined lenvatinib and immunotherapy. ${ }^{18}$ However, there are currently no data indicating the optimal strategy for conversion hepatectomy such as when to initiate surgery after an initial response to systemic therapy. In this article, we report the case of a patient with recurrent advanced HCC who achieved downstaging following third-line treatment with lenvatinib, but delayed and missed an opportunity for conversion hepatectomy. Written, informed consent to publish details and images from this case was obtained from the patient.

\section{Case Presentation}

A male Chinese patient aged 42 years with a height of $193 \mathrm{~cm}$, weight of $95 \mathrm{~kg}$ and a history of chronic hepatitis $B$ infection for $>20$ years. The patient presented with an obstructive liver lesion, as assessed by physical examination, and was admitted to hospital for 2 weeks. Computed tomography (CT) imaging evaluation identified a single tumor with diameter approximately $3 \mathrm{~cm}$ located in segments V and VIII of the liver without macrovascular invasion. The tumor was staged as TNM stage T1bN0M0 $\mathrm{Ib}$, corresponding to Barcelona Clinic Liver Cancer (BCLC) Stage A. The patient had an Eastern Co-operative Oncology Group Performance Score (ECOG PS) of 0, no jaundice of the skin or sclera, and negative abdominal signs. Laboratory tests revealed that the patient had normal liver function (Child-Pugh Score 5 and Grade A) and coagulation, an AFP level $4.45 \mathrm{ng} / \mathrm{mL}$, hepatitis B surface antigen titer of 1816 , HBV DNA level of $3.45 \times 10^{4} \mathrm{IU} / \mathrm{mL}$ and Protein Induced by Vitamin K Absence or AntagonistII (PIVKA-II) level $34 \mathrm{mAU} / \mathrm{mL}$. Based on the single liver lesion, early stage of disease, good liver function and discussion with the patient, a hepatectomy was performed.

After a successful hepatectomy with a surgical margin of $1 \mathrm{~cm}$ to the tumor, pathological examination revealed the tumor had a diameter of approximately $3 \mathrm{~cm}$ with a negative surgical margin consisting of moderately differentiated HCC and without tumor thrombus or satellite foci. After surgery, the patient received one cycle of TACE to identify residual lesions and confirm successful resection, and no residual tumor was observed. Post-surgery, the patient received thymosin to prevent the relapse of $\mathrm{HCC}$, as well as oral entecavir (500 mg/day) for the management of hepatitis B. The patient experienced tumor recurrence 14 months after surgery with a single tumor of approximately $2 \mathrm{~cm}$ diameter located between the left and right hepatic pedicle of the first porta hepatis. The tumor was close to the portal vein and biliary tract without macrovascular involvement. The recurrent tumor was early stage (T1aN0M0 Ia) and based on the Milan criteria the patient was a potential candidate for liver transplantation. However, the patient refused to undergo liver transplantation and underwent a CT-guided microwave ablation procedure. Post-surgery TACE revealed complete tumor ablation without residual tumor. The patient continued to 
receive thymosin to prevent $\mathrm{HCC}$ recurrence, as well as oral entecavir for hepatitis B.

After a further 11 months, the patient experienced a second recurrence characterized by multiple disseminated metastases in the left and right lobes of the liver and possible macrovascular invasion. No distant metastasis was identified and the patient had an ECOG PS of 0 with Child Pugh Stage 5 and Grade A. However, the tumor stage of the patient was relatively advanced beyond transplantation criteria (BCLC Stage B/C) (Figure 1A).
Based on guideline recommendations, the patient was advised to initiate systemic therapy. ${ }^{3,11}$ Genetic testing indicated a low tumor mutation burden and immunohistochemical analysis was negative for programmed cell death ligand 1 (PD-L1) expression. A microsatellite stability test found no variation for MLH1, MSH2, MSH6, and PMS2, which indicated a low probability of benefit from immune therapy and this was communicated to the patient. The patient therefore initiated treatment with lenvatinib (Lenvima $^{\circledR}$, Eisai Co., Japan) $12 \mathrm{mg}$ once daily and
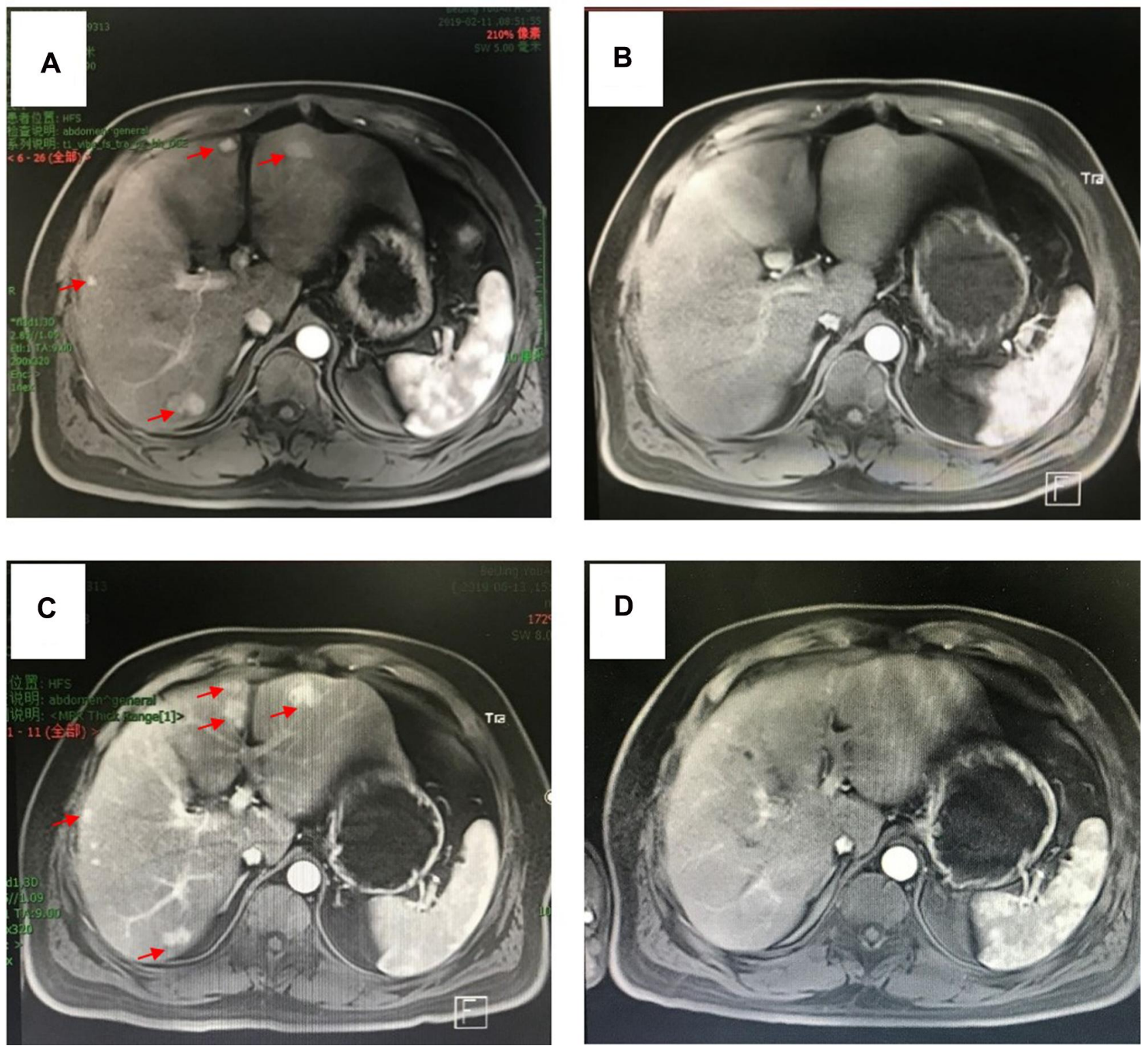

Figure I Representative magnetic resonance imaging data in the late arterial phase showing the clinical course of the patient. (A) Before systemic therapy the patient had BCLC Stage B/C HCC characterized by multiple disseminated metastases in the left and right lobes of the liver and possible macrovascular invasion (indicated by red arrows); (B) after 2 months of lenvatinib treatment the patient achieved a partial remission (mRECIST criteria); (C) after one month of glucocorticoid therapy and switching from lenvatinib to apatinib the patient experienced progressive disease as evaluated by imaging examination (recurrent disease indicated by red arrows); (D) after switching back to lenvatinib the patient achieved a partial response, with tumor stage recovered to BCLC Stage $A$. 
achieved a partial remission (mRECIST criteria) by imaging evaluation after 3 months of treatment (Figure 1B), and a reduction in PIVKA-II levels from 254 to $142 \mathrm{mAU} /$ $\mathrm{mL}$. Three months after initiation of lenvatinib, the patient decided to transfer to another hospital (Peking Union Medical College Hospital) with specialized expertise in the use of anti-programmed cell death 1 (PD-1) antibody therapy, at which they could begin additional treatment with anti-PD-1 antibodies (off label, toripalimab, Shanghai Junshi Biosciences, Shanghai, China) (240 $\mathrm{mg}$ ). The patient experienced a severe immune reaction (Stevens-Johnson syndrome) 6 days after initiating immune therapy. Subsequently, the patient immediately terminated PD-1, and after returning to our institution received intravenous glucocorticoid therapy for one month, before switching from lenvatinib to apatinib monotherapy (off-label, Aitan ${ }^{\circledR}$, Jiangsu Hengrui Medicine, China) (850 mg daily) for 10 days. The patient was then found to have progressive disease as evaluated by imaging examination (Figure 1C), accompanied by an increase in PIVKA-II level from 142 to $1019 \mathrm{mAU} / \mathrm{mL}$. After multidisciplinary consultation, the patient was switched back to lenvatinib monotherapy.

After one month of lenvatinib treatment, the patient was found to have again achieved a partial response (Figure 1D). In addition, the patient's PIVKA-II level had decreased from 1019 to $324 \mathrm{mAU} / \mathrm{mL}$ and the tumor stage recovered to BCLC Stage A. Based on the Milan criteria the patient was suitable for a liver transplantation and was advised to make the most of this "window of opportunity". However, the patient refused surgery. After being in remission for 4 months, the patient's PIVKA-II level increased, which was defined as progressive disease. The tumor stage of the patient advanced to BCLC C and the patient missed the opportunity for radical hepatectomy. At this stage, the patient was switched from lenvatinib to regorafenib $80 \mathrm{mg}$ daily. The patient was followed up for the last time on April 21, 2020, and PET-CT revealed bone and lung metastasis (Figure 2).

\section{Discussion}

For patients with $\mathrm{HCC}$, surgical interventions including hepatectomy and liver transplantation provide the best
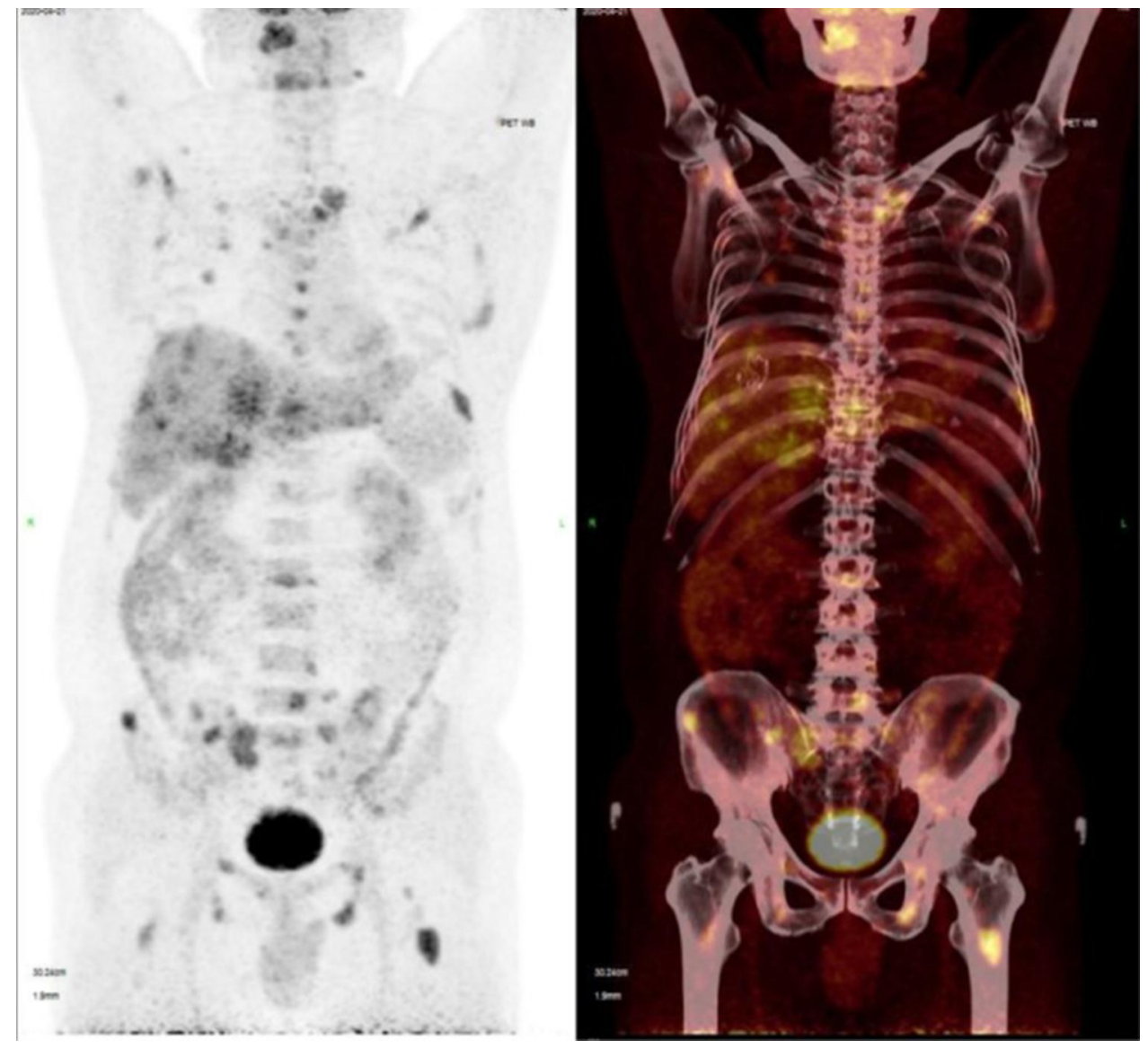

Figure 2 Representative PET-CT imaging showing bone and lung metastasis. 
long-term survival outcomes. ${ }^{2-6}$ However, only a minority of patients with HCC are diagnosed with early or moderate disease suitable for surgical intervention. ${ }^{3,8}$ Particularly in China, the majority of patients present with advanced or metastatic HCC and are only eligible for locoregional or systemic therapy, which are associated with modest survival benefits. ${ }^{19}$ Despite this situation, the pivotal Phase III trial of lenvatinib revealed a particularly high ORR (40.6\%), suggesting an opportunity for downstaging and broadening access to curative surgical interventions among patients with advanced HCC. ${ }^{13}$ In this regard, our case study reports a patient with recurrent, advanced HCC who achieved a partial response following treatment with lenvatinib, leading to sufficient downstaging to enable surgical intervention. Unfortunately, the patient refused surgery and ultimately experienced progressive disease. Our case highlights the need for evidence-based strategies for the management of patients with HCC who achieve a good response to systemic therapy and become potential candidates for surgical intervention.

In recent years, targeted anti-cancer drugs including sorafenib and lenvatinib have shown efficacy against HCC in a number of large Phase III trials. ${ }^{9-11}$ Combination treatment strategies combining targeted therapies with immune checkpoint inhibitors have also achieved breakthrough progress, with the potential to provide additional survival benefits for patients with advanced HCC. ${ }^{14-16}$ Results from the Phase III REFLECT trial demonstrated that lenvatinib is non-inferior to sorafenib for overall survival in patients with advanced HCC and is also associated with the highest ORR reported for any systemic therapy in HCC (up to $40.6 \%$ ). ${ }^{13}$ This high response rate may be related to the mode of action of lenvatinib, which targets multiple tyrosine kinases associated with tumor growth, angiogenesis, and progression including VEGFR1-3, FGFR1-4, PDGFR, RET and KIT. Importantly, the high ORR associated with lenvatinib means that a considerable proportion of patients will achieve reductions in tumor size, decreased tumor load and even decreased tumor stage. This provides an opportunity for patients with initially unresectable HCC to achieve conversion to radical hepatectomy.

There have been multiple case reports of successful conversion hepatectomy in patients with advanced HCC following lenvatinib or combination treatment with lenvatinib and immune therapy. One case reported a patient who presented with a large $(14.5 \times 10.9 \mathrm{~cm}) \mathrm{HCC}$ tumor and was initially unsuitable for hepatectomy. After receiving lenvatinib $8 \mathrm{mg}$ daily plus anti-PD-1 antibodies (nivolumab; $140 \mathrm{mg}$ IV every 2 weeks for 6 months) the patient successfully underwent an extended right hepatectomy. ${ }^{18}$ A further report described a similar case of a patient presenting with a very large (diameter $10 \mathrm{~cm}$ ) $\mathrm{HCC}$ tumor and poor liver function who was considered unsuitable for surgical resection. ${ }^{20}$ The patient failed initial treatment with TACE due to the presence of a major arterioportal shunt, but was then successfully treated with lenvatinib $8 \mathrm{mg}$ daily for 4 months followed by successful TACE treatment. The patient responded well to treatment and regained sufficient liver function to enable a successful conversion hepatectomy. Taken together with these previously published cases, the present case suggests that tumor downstaging through combinations of locoregional therapy, lenvatinib and immune therapy can enable patients with advanced $\mathrm{HCC}$ to receive potentially curative surgical resection.

Although downstaging strategies have the potential to greatly benefit patients with $\mathrm{HCC}$, there remain a number of questions and challenges. Firstly, for patients who achieve a complete or partial response to therapy and become eligible candidates for conversion hepatectomy, is it currently unclear how to decide between continuation of systemic therapy and surgical intervention. Secondly, the optimal timing between achievement of a response to systemic therapy and conversion hepatectomy is unclear, and is the key "pitfall" of this treatment strategy. Achieving a remission during systemic therapy can be misunderstood by patients as an indication their cancer has been controlled or eradicated. In this regard, treatment with targeted drugs can lead to relatively good long-term survival but negate the opportunity for achieving optimal survival through radical treatment. In the Phase III trial of lenvatinib in advanced $\mathrm{HCC}$, the median progression-free survival (PFS) was 8.9 months (95\% CI; 7.4-9.2). ${ }^{13}$ Therefore, the "window of opportunity" for conversion hepatectomy following a response to lenvatinib could be estimated to lie within this timeframe. Once patients experience disease progression, they will lose the chance for potentially curative surgical intervention. This is well illustrated in our case, in which the patient achieved a partial response to lenvatinib but experienced tumor progression after 4 months, progressing to BCLC Stage $\mathrm{B} / \mathrm{C}$ and losing the opportunity for radical hepatectomy. This suggests that patients who achieve downstaging during lenvatinib treatment and become suitable for radical 
hepatectomy should undergo surgery within the estimated PFS timeframe.

\section{Conclusion}

A patient with recurrent advanced HCC achieved a partial response and downstaging following third-line lenvatinib treatment, but ultimately lost the opportunity to undergo radical hepatectomy due to rapid disease progression. This case suggests that patients achieving downstaging during systemic therapy should undergo conversion hepatectomy in a timely manner and within the estimated PFS associated with the systemic therapy being used. However, further studies are required to provide additional evidence for this treatment strategy.

\section{Abbreviations}

BCLC, Barcelona Clinic Liver Cancer; ECOG PS, Eastern Co-operative Oncology Group Performance Score; HCC, hepatocellular carcinoma; ORR, objective response rate; PIVKA-II, Protein Induced by Vitamin K Absence or Antagonist-II; PD1, programmed cell death 1; PD-L1, programmed cell death ligand 1; PFS, progression-free survival; TACE, transarterial chemoembolization.

\section{Ethics Approval and Informed Consent}

Informed consent was obtained from the patient before submission of this case study. Institutional ethical approval for publication of this case study was not required.

\section{Consent for Publication}

Informed consent was obtained from the patient before submission of this case study.

\section{Acknowledgments}

Editorial support for this manuscript was provided by Jake Burrell, PhD CMPP (Rude Health Consulting Shanghai).

\section{Author Contributions}

All authors made substantial contributions to conception and design, acquisition of data, or analysis and interpretation of data; took part in drafting the article or revising it critically for important intellectual content; agreed on the journal to which the article will be submitted; gave final approval of the version to be published; and agree to be accountable for all aspects of the work.

\section{Funding}

No funding was received for this study.

\section{Disclosure}

The authors have no conflicts of interest to declare.

\section{References}

1. Bray F, Ferlay J, Soerjomataram I, Siegel RL, Torre LA, Jemal A. Global cancer statistics 2018: GLOBOCAN estimates of incidence and mortality worldwide for 36 cancers in 185 countries. CA Cancer J Clin. 2018;68(6):394-424.

2. Chawla A, Ferrone C. Hepatocellular carcinoma surgical therapy: perspectives on the current limits to resection. Chin Clin Oncol. 2018;7(5):48. doi:10.21037/cco.2018.08.12

3. Llovet JM, Bru C, Bruix J. Prognosis of hepatocellular carcinoma: the BCLC staging classification. Semin Liver Dis. 1999;19(3):329338. doi:10.1055/s-2007-1007122

4. Llovet JM, Fuster J, Bruix J. Intention-to-treat analysis of surgical treatment for early hepatocellular carcinoma: resection versus transplantation. Hepatology. 1999;30(6):1434-1440. doi:10.1002/ hep. 510300629

5. Chok KS, Ng KK, Poon RT, Lo CM, Fan ST. Impact of postoperative complications on long-term outcome of curative resection for hepatocellular carcinoma. Br J Surg. 2009;96(1):81-87. doi:10.1002/ bjs. 6358

6. Kianmanesh R, Regimbeau JM, Belghiti J. Selective approach to major hepatic resection for hepatocellular carcinoma in chronic liver disease. Surg Oncol Clin $N$ Am. 2003;12(1):51-63. doi:10.1016/S1055-3207(02)00090-X

7. Siegel RL, Miller KD, Jemal A. Cancer statistics, 2019. CA Cancer J Clin. 2019;69(1):7-34.

8. Forner A, Reig M, Bruix J. Hepatocellular carcinoma. Lancet. 2018;391(10127):1301-1314. doi:10.1016/S0140-6736(18)30010-2

9. Galle PR, Forner A, Llovet JM, European Association for the Study of the Liver. Electronic address eee, European Association for the Study of the L. EASL clinical practice guidelines: management of hepatocellular carcinoma. $J$ Hepatol. 2018;69(1):182-236. doi:10.1016/j.jhep.2018.03.019

10. Omata M, Cheng AL, Kokudo N, et al. Asia-Pacific clinical practice guidelines on the management of hepatocellular carcinoma: a 2017 update. Hepatol Int. 2017;11(4):317-370.

11. Network NCC. National comprehensive cancer network. Hepatobiliary cancers (version 3.2018). 2018. Available from: https://www.nccn.org/professionals/physician_gls/pdf/hepatobiliary. pdf. Accessed October, 2018.

12. Llovet JM, Ricci S, Mazzaferro V, et al. Sorafenib in advanced hepatocellular carcinoma. $N$ Engl J Med. 2008;359(4):378-390. doi:10.1056/NEJMoa0708857

13. Kudo M, Finn RS, Qin S, et al. Lenvatinib versus sorafenib in firstline treatment of patients with unresectable hepatocellular carcinoma: a randomised Phase 3 non-inferiority trial. Lancet. 2018;391 (10126):1163-1173. doi:10.1016/S0140-6736(18)30207-1

14. Kudo M. Extremely high objective response rate of lenvatinib: its clinical relevance and changing the treatment paradigm in hepatocellular carcinoma. Liver Cancer. 2018;7(3):215-224.

15. Liu Z, Li X, He X, Xu Y, Wang X. Complete response to the combination of Lenvatinib and Pembrolizumab in an advanced hepatocellular carcinoma patient: a case report. BMC Cancer. 2019;19 (1):1062. doi:10.1186/s12885-019-6287-8

16. Ikeda M, Sung MW, Kudo M, et al. Abstract CT061: a phase Ib trial of lenvatinib (LEN) plus pembrolizumab (PEMBRO) in unresectable hepatocellular carcinoma (uHCC): updated results. Cancer Res. 2019;79(13Supplement):CT061-CT061. 
17. Fujita M, Okai K, Hayashi M, et al. Huge hepatocellular carcinoma treated with radical hepatectomy after drug-eluting bead transarterial chemoembolization. Intern Med. 2019;58(8):1103-1110. doi:10.2169/internalmedicine.1214-18

18. Chen X, Zhang Y, Zhang N, Ge Y, Jia W. Lenvatinib combined nivolumab injection followed by extended right hepatectomy is a feasible treatment for patients with massive hepatocellular carcinoma: a case report. Onco Targets Ther. 2019;12:7355-7359. doi:10.2147/OTT.S217123
19. Chen W, Zheng R, Baade PD, et al. Cancer statistics in China, 2015. CA Cancer J Clin. 2016;66(2):115-132.

20. Sato N, Beppu T, Kinoshita K, et al. Conversion hepatectomy for huge hepatocellular carcinoma with arterioportal shunt after chemoembolization and lenvatinib therapy. Anticancer Res. 2019;39 (10):5695-5701.

\section{Publish your work in this journal}

OncoTargets and Therapy is an international, peer-reviewed, open access journal focusing on the pathological basis of all cancers, potential targets for therapy and treatment protocols employed to improve the management of cancer patients. The journal also focuses on the impact of management programs and new therapeutic

Submit your manuscript here: https://www.dovepress.com/oncotargets-and-therapy-journal agents and protocols on patient perspectives such as quality of life, adherence and satisfaction. The manuscript management system is completely online and includes a very quick and fair peer-review system, which is all easy to use. Visit http://www.dovepress.com/ testimonials.php to read real quotes from published authors. 